\title{
Fuzzy Logic Attitude Control System for a Mini Helicopter Expanded Non Linear Mathematical Model
}

\author{
Abdul Ghafoor Al-Shehabi \\ Department of Mechanical Engineering, University of Alberta, Edmonton, Canada
}

\begin{abstract}
Full nonlinear mathematical model for a mini helicopter is very complex, inflexible and has big influence on the designed control system performance. Analytical approach for autonomous helicopter modeling including both swash plate and mixer dynamics additional to miniature helicopter equations of motion is introduced. Attitude control system without linearization process is directly designed using fuzzy logic concepts, fuzzy controllers are implemented in both stability and command augmentation loops. Scaling factors are adjusted to minimize the error. The performance of control system for miniature helicopter expanded nonlinear model using fuzzy logic controllers is evaluated through simulation.
\end{abstract}

Keywords: Flight control, fuzzy logic, UAV, helicopter, swashplate.

\section{Introduction}

Small-scale unmanned aerial vehicles are widely used in many application fields because of their small size and superior flight characteristics; they have gained much attention and become an ideal platform for academic research [1], they have the same characteristics of full-scale rotorcraft and own some unique and attractive features such as low cost, easy operation, and extreme agility [2]. The miniature helicopter have been developed to be capable of working autonomously without interference of a human pilot for the purposes of scientific observations, detecting disasters, surveillance of traffic and army objectives [3].

Miniature helicopter models are characterized by strongly coupled, multi-varieties and time delay nonlinear systems [4], therefore the design of an elaborated control system for autonomous flight is a challenging task. Flight control system design is considered one of the core issues in the development of a fully functional unmanned helicopter, many control system designs have been developed for controlling

Corresponding author:Abdul Ghafoor Al-Shehabi, professor, visiting professor in the Mechanical Engineering Department, University of Alberta, Edmonton, Canada. the dynamic process of miniature helicopter; few among those designs are readily suitable for applying [5]. Several controller design methods are successfully implemented as control technologies such as the adaptive control [6,7], optimal control [8], neural network approach $[9,10]$, the robust and $\mathrm{H}_{\infty}$ control approach [11], and fuzzy logic [12]. However, many of these reported works have one common drawback, they focus merely on the basic miniature helicopter equations of motion without including both the swashplate and mixer mechanism dynamics within miniature helicopter mathematical model, so the efficiency of control system is limited. The controller design methods used in these works rely on miniature helicopter dynamical equations of motions that have four indirect primary input commands $U_{c o l}, U_{\text {lon }}, U_{\text {lat }}$ and $U_{\text {ped }}$ for controlling vertical velocity, the pitch, roll, and yaw rate, respectively [13]. This paper deals with challenging modeling, control and simulation processes of miniature helicopter due to the designing attitude control system for miniature helicopter including swash plate and mixer dynamics within its nonlinear model.

The need to use real vehicles in the control design cycle poses a high risk and it is prohibitively high cost. To overcome this problem, a real time simulation 
concept that employs cheap, practical, and rapid-to-build modular hardware, which can simulate a nearly real processin lab's environment is adopted. Also, it is very difficult to develop an autonomous flight control system of unmanned helicopter based on the conventional method because the dynamic of miniature helicopter is usually highly nonlinear and contains many uncertainties. In order to overcome these difficulties, one of the intelligent control theories has been investigated [14]. Fuzzy logic concepts are gracefully employed in unmanned helicopter control system design, the flight control system designed based on fuzzy logic concepts can be performed based on miniature helicopter nonlinear mathematical models at different reference flight conditions. PD(Proportional-derivative)fuzzy controller for each control variable has been used in the flight control system design because of its simple structure and its robust performance, the controller has first to measure the miniature helicopter actual output and compare it with the desired one in order to generate the control signal, so the controller output depends on the actual error and short part of the past state trajectory. The aim of this work is to design an intelligent flight control system for small-scale unmanned helicopter in order to adjust the desired attitude performance. To realize this goal, nonlinear mathematical model including the dynamic of actuators for mini helicopter is illustrated, the flight control system with two hierarchical layers, in which the inner-loop layer is used for stabilizing the helicopter model, while the outer-loop layer is used for controlling its attitude angles, is carried out. For each control loop, the designed MISO(multi-inputs single outputs) controllers in all its sub-systems are blended to form MIMO(multi-inputs multi outputs) fuzzy controller [15]. This paper elaborates the integration of nonlinear mathematical model for small scale helicopter, with swash plate and mixer dynamics in addition to wind models into a modular model.

This paper is organized as follows. Section 2 provides the motivation for the work presented in this paper by discussing the need for implementing and using fuzzy logic concepts in flight control system design. Section 3 describes the nonlinear mathematical model of the mini helicopter and other dynamical subsystems to generate the input variables of unmanned helicopters nonlinear mathematical model. Section 4 presents the overall cascaded control scheme used to perform the control tasks to be executed using both inner-loop and outer-loop controllers. Fuzzy logic controllers of Mamdani type are designed in both inner and outer loops; the outer-loop controllers are blended to be able to adjust the attitude angles at desired values, while the inner-loop controllers are blended to adjust the required angular velocity components that will result in that attitude. Section 5 presents the simulation results showing that the objective of using fuzzy logic controller to adjust the attitude angles has been achieved. In Section 6 some conclusions and future works are provided to the readers.

\section{Non-linear Mathematical Model}

In order to successfully design the control system of unmanned aerial helicopter, it will be considered as a rigid body, so its mathematical model generally can be derived and developed based on either Newton's laws of motion or the Euler-Lagrange equation for motion [16]. As it is shown in Fig. 1, the body coordinate system is defined with the origin at the center of gravity of the miniature helicopter. The $\mathrm{x}$-axis is pointing to the nose of the miniature helicopter and it is positive in the forward direction, the y-axis is positive to the right, to be matched with the right-handed concepts used as usual convention for the analysis of stability and control, the z-axis coincides with the orientation of the principal rotor axis and it is positive downwards. The miniature helicopter has mass $m$ and moments of inertia $I_{x x}, I_{y y}$, and $I_{z z}$, on the roll, pitch, and yaw axis respectively. Both the mass of the rotor and the blade moments of 


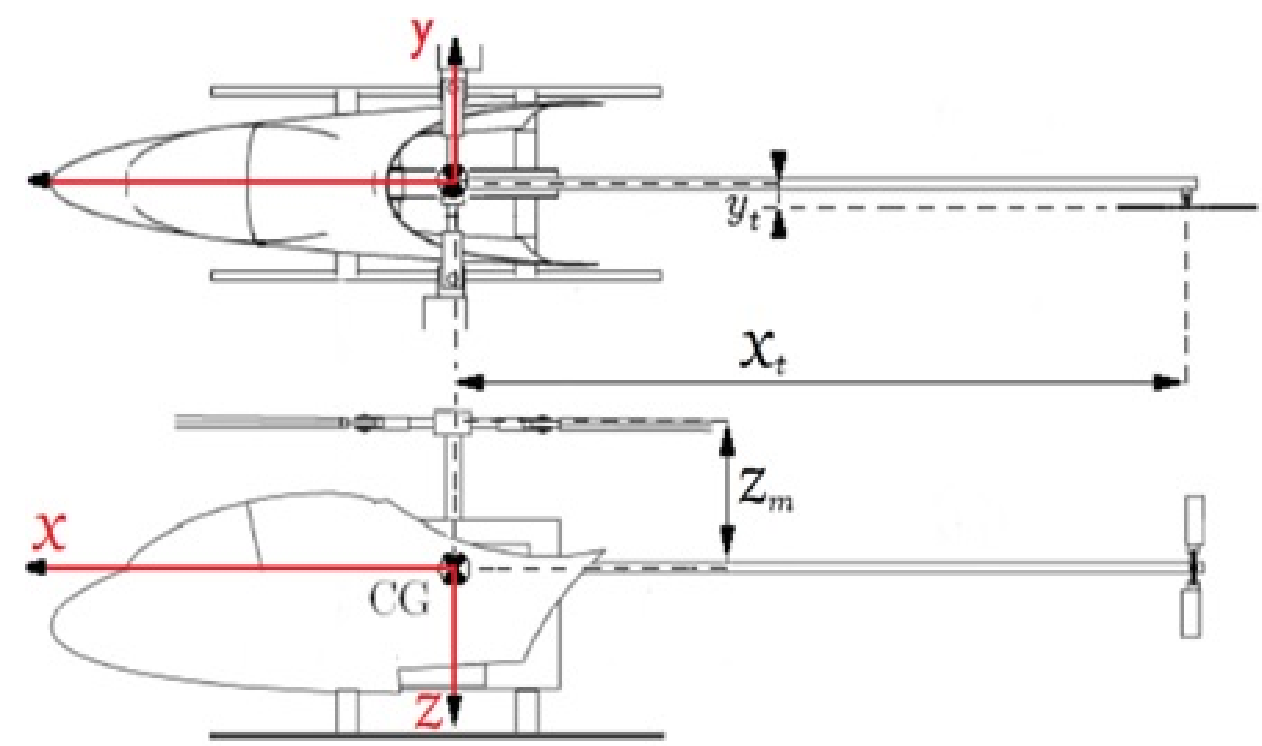

Fig. 1 Helicopter principal axis.

inertia $I_{b}$ are also implemented in miniature helicopter nonlinear mathematical model. There is a plane of symmetry along the miniature helicopter zx-axis. Thus, $I_{z x}=0$; there is a plane of symmetry of mass along the yz-axis on the helicopter, this implies $I_{y z}=0$. The miniature helicopter technical characteristic data used in modeling and simulation are matched with real model available at university of Alberta. Recently, many related parameters are extracted and identified [17-18].

The movement of miniature helicopter is achieved either by changing the pitch of all the main rotor blades, collective pitch, thus increasing rotor thrust, or by changing the pitch as a sinusoidal function of azimuth, cyclic pitch, which tilts the tip-path-plane fore/aft or left/right and changes the thrust vector direction, also the movement is achieved by changing the tail rotor pitch, which changes tail rotor thrust and thus it greats the yaw moment. These resulted forces and moments must simultaneously be adjusted, hence the control of miniature helicopter is a difficult task indeed. The longitudinal control makes the miniature helicopter rotates about the y-axis and thereby it performs a pitch moment, while the lateral control makes the miniature helicopter to rotate about the $\mathrm{x}$-axis and thereby it performs a roll moment. Both lateral and longitudinal control form the cyclic pitch control input, the cyclic inputs produce rotor moments and then change the fuselage attitude, but they cannot control the miniature helicopter's position and velocity directly, After the fuselage's roll and pitch angles are changed, the rotor thrust is tilted accordingly to produce horizontal projections as propulsive forces. In this way, the miniature helicopter's translational states can be controlled. The vertical movement of miniature helicopter is performed by the amount of thrust generated by the main rotor collective pitch. The directional control makes the miniature helicopter rotates about the z-axis and thereby it performs a yaw moment. The tail rotor thrust direction is considered as opposite to the main rotor thrust direction. The main rotor $\operatorname{speed} \Omega$ is maintained constant by a so called governor to reduce the number of control inputs. Typically, a cyclic stick is used to control both fore/aft motions, pitch control and left/right motion, roll control, and collective lever is used to control up and down motions, vertical control, while the pedals are used to control left and right yawing motions, yaw control, so five electromechanical servos, controlled by PWM (Pulse Width Modulation) signals are required to actuate the miniature helicopter model. 


\subsection{Rigid Body Model}

Generally, the mini helicopter has eight-degree-of-freedom in its motion, which is composed of translational displacements and rotational motions: up/down, fore/aft as longitudinal motion, left/right as lateral motion, pitching, rolling, and yawing, additional to two main rotor flapping angles, $a$ and $b$. The miniature helicopter has nine basic state variables in general, which are the velocity components at center of gravity, they are $U, V$ and $W$, along body reference axes, three angular rates $P, Q$ and $R$, the aircraft roll, pitch and yaw rates about body reference axes, and three attitude angles $\Phi, \Theta$ and $\Psi$ known as Euler angles which define the orientation of the fuselage with respect to earth axes system [19]. There exist two reference frames which are generally used to determine the motion of miniature helicopter; the body-fixed framework and the inertial framework. The non-linear equations of motion with respect to the body-fixed reference frame can be written as

$$
\begin{aligned}
& \dot{U}=V R-W Q-G \sin \Theta+F_{x} / m \\
& \dot{\mathrm{V}}=\mathrm{W} \mathrm{P}-\mathrm{UR}+\mathrm{G} \sin \Phi \cos \Theta+\mathrm{F}_{\mathrm{y}} / \mathrm{m} \\
& \dot{\mathrm{W}}=\mathrm{U} \mathrm{Q}-\mathrm{V} \mathrm{P}+\mathrm{G} \cos \Phi \cos \Theta+\mathrm{F}_{\mathrm{z}} / \mathrm{m} \\
& \dot{\mathrm{P}}= \\
& \frac{\mathrm{L}-2 \mathrm{Q}\left\{-\mathrm{Iz}_{\mathrm{b}} \Omega+\mathrm{r}\left[\left(\mathrm{Izz}+\mathrm{Iz}_{\mathrm{b}}\right)-\left(\mathrm{Iyy}+\frac{\mathrm{Iz}_{\mathrm{b}}+\mathrm{Iz}_{\mathrm{b}}}{2}\right)\right]\right\}}{2 \mathrm{Ixx}+\mathrm{Ix}_{\mathrm{b}}+\mathrm{Iy}_{\mathrm{b}}} \\
& \dot{Q}= \\
& \underline{M-2 P\left\{I z_{b} \Omega+r\left[\left(I x x+\frac{I x_{b}+I y_{b}}{2}\right)-\left(I z z+I z_{b}\right)\right]\right\}} \\
& 2 \mathrm{Iyy}+\mathrm{Ix}_{\mathrm{b}}+\mathrm{Iy}_{\mathrm{b}} \\
& \dot{\mathrm{R}}=\frac{\mathrm{N}+\mathrm{P} Q(\mathrm{Ixx}-\mathrm{Iyy})}{\mathrm{Izz}+\mathrm{Iz}_{\mathrm{b}}} \\
& \dot{\Phi}=\mathrm{P}+\mathrm{Q} \sin \Phi \tan \Theta+\mathrm{R} \mathrm{Q} \cos \Phi \tan \Theta \\
& \dot{\Theta}=Q \cos \Phi-R \sin \Phi \\
& \dot{\Psi}=\mathrm{Q} \frac{\sin \Phi}{\cos \Theta}+\mathrm{R} \frac{\cos \Phi}{\cos \Theta}
\end{aligned}
$$

where, $\vec{F}=\left[\begin{array}{lll}F_{x} & F_{y} & F_{z}\end{array}\right]^{T}$ is the vector of external forces acting on the helicopter c.g., and $\vec{M}=$ $\left[\begin{array}{lll}L & M & N\end{array}\right]^{T}$ is the vector of external momentsin body-coordinate system.

\subsection{Swash Plate and Mixer Dynamics}

Swashplate mechanism is an important part in almost all layouts of helicopters. The main function of this mechanism is to provide an applicable way for adjusting the blade pitch angles of the main rotor, either via the collective control or via the cyclic control, according to given control input commands represented by rotation angles of the servos [20]. The swash plate consists of two plates and various control rods. The lower plate is fixed to the main miniature helicopter body whereas the upper plate is rotating with the main rotator drive shaft. The rods that are connected to the lower plate are affected by the cyclic and collective controls, thus changing the inclination of the whole plate to various directions according the control inputs, see Fig. 2. The inclination of the lower plate is transferred to the upper plate through a set of bearings between the two plates, which allow the upper rotating plate to spin on top of the lower non-rotating plate. The inclination of swashplate affects the position of the upper control rods that are responsible for the angle of attack of the rotor blades. The values of PW(Pulse Width) calculated from roll, pitch, and collective commands are typically governed by the type of swashplate on the vehicle. Most commercial mini helicopters have either a three or four points swashplate. These points represent the number of servo arms that controls the position and orientation of the swashplate. Four points swash plates are by far the most common and they are the easiest to comprehend. Typically, in a four point system, there are dedicated servos for lateral commands, roll, longitudinal commands, pitch, and collective commands. The swash plate used in the proposed mini helicopter is from 4-point linkages type, so four servos control the three dimensional swash plate motion, they directly define both two swash plate rotations and one 


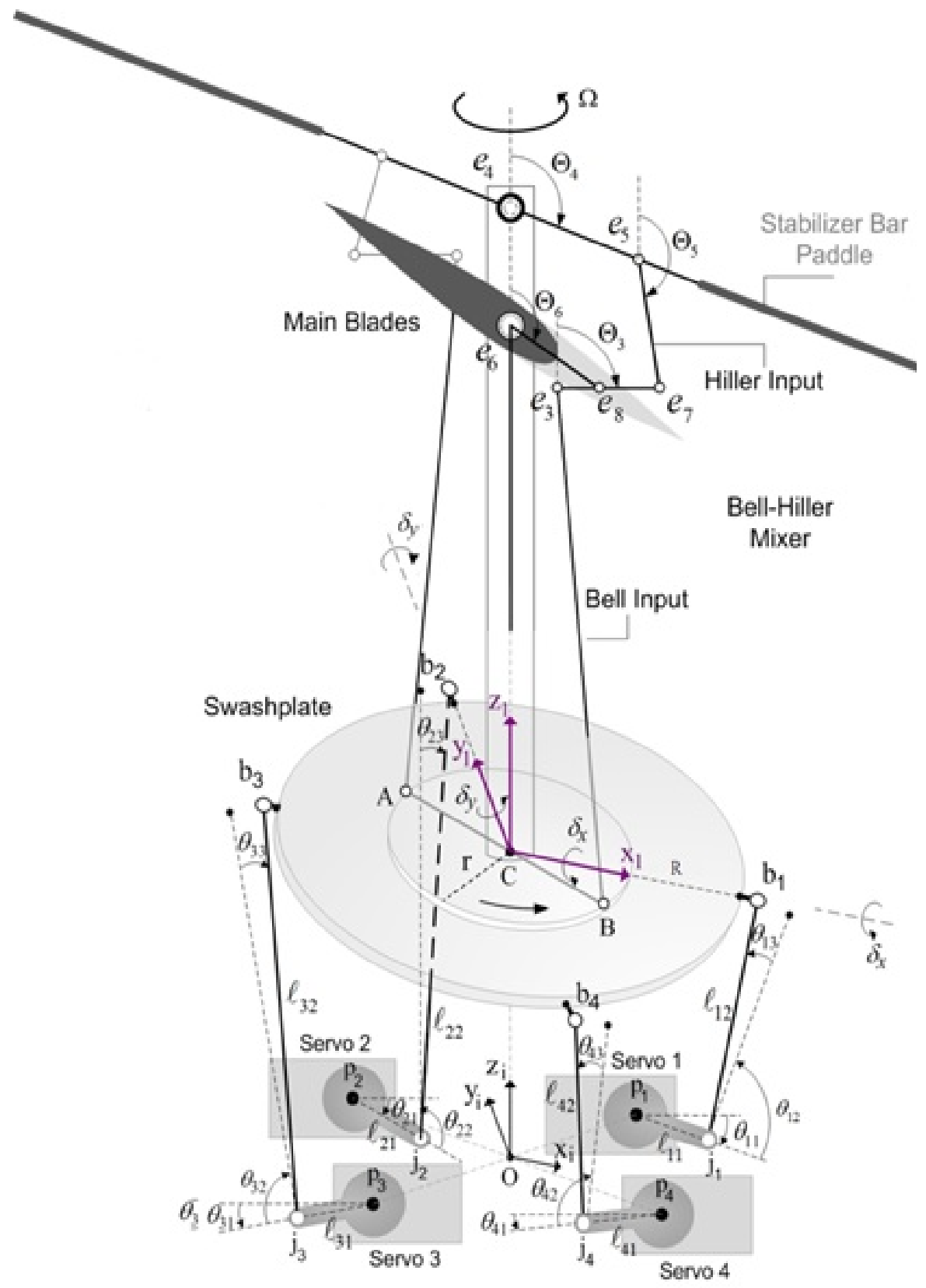

Fig. 2 Swash plate and mixer mechanism.

swash plate translation, thus they are indirectly controlling the pitch angle of the blades of the main rotor. All used servos are identical and their saturation limits are considered, the systematic and intensive study of actuator mechanism performance adds considerable contribution to the modeling and control of the mini helicopter.

The swash plate dynamic can be modeled mathematically through four nonlinear and coupled equations of motion, each swash plate equation of motion is related to one of the contributed servos represented by its rotation angle $\Theta_{i 1}$. The longitudinal or lateral rotation of swash plate can be produced by commending two opposite servos to rotate in the same rotation angle but in opposite rotation direction, while the vertical displacement of swash plate can be produced by commending all servos to rotate in the same value and direction of rotation angles. According 
to the swash plate and mixer mechanism shown in Fig. 2, the mathematical model of swash plate can be derived and be represented by the following four nonlinear and coupled equations.

$$
\begin{gathered}
{\left[R \cos (\delta y)-x_{p 1}-l_{11} \cos \left(\theta_{11}\right)\right]^{2}+[R \sin (\delta y)-} \\
\mathrm{zc}-\ell 1 \sin \theta 112-1-y p 11112112=0 \\
{\left[\mathrm{R} \sin (\delta \mathrm{x}) \sin (\delta \mathrm{y})-\mathrm{x}_{\mathrm{p} 2}-\mathrm{l}_{21} \cos \left(\theta_{21}\right)\right]^{2}+} \\
{\left[-\mathrm{R} \sin (\delta \mathrm{x}) \cos (\delta \mathrm{y})-\mathrm{zc}-\mathrm{l}_{21} \sin \left(\theta_{21}\right)\right]^{2}-} \\
{\left[1-\left(\frac{\mathrm{R} \cos (\delta \mathrm{x})-\mathrm{y}_{\mathrm{p} 2}}{\mathrm{l}_{21}}\right)^{2}\right] \mathrm{l}_{21}{ }^{2}=0} \\
{\left[\mathrm{R} \cos (\delta \mathrm{y})+\mathrm{x}_{\mathrm{p} 3}-\mathrm{l}_{31} \cos \left(\theta_{31}\right)\right]^{2}+} \\
{\left[\mathrm{R} \sin (\delta \mathrm{y})+{ }^{2}-\left[1-\left(\frac{\mathrm{y}_{\mathrm{p} 3}}{\mathrm{l}_{31}}\right)^{2}\right] \mathrm{l}_{31}{ }^{2}=0\right.} \\
\left.\mathrm{zc}-\mathrm{l}_{31} \sin \left(\theta_{31}\right)\right] \\
{\left[\mathrm{R} \sin (\delta \mathrm{x}) \sin (\delta \mathrm{y})+\mathrm{x}_{\mathrm{p} 4}-\mathrm{l}_{41} \cos \left(\theta_{41}\right)\right]^{2}+} \\
{\left[-\mathrm{R} \sin (\delta \mathrm{x}) \cos (\delta \mathrm{y})+\mathrm{zc}^{2}-\mathrm{l}_{41} \sin \left(\theta_{41}\right)\right]^{2}-} \\
{\left[1-\left(\frac{\mathrm{R} \cos (\delta \mathrm{x})+\mathrm{y}_{\mathrm{p} 4}}{\mathrm{l}_{41}}\right)^{2}\right] \mathrm{l}_{41}{ }^{2}=0}
\end{gathered}
$$

The input variables in swash plate mathematical model are represented by the rotation angles of four servos, they are $\Theta_{11}, \Theta_{21}, \Theta_{31}$ and $\Theta_{41}$, while the output variables are represented by swash plate longitudinal rotation angle $\delta y$, lateral rotation angle $\delta x$ and vertical displacement $z c$. The other variables in swash plate equations have constant values, they are swash plate radius $R$, the lengths $l_{i j}$ of connecting rods and the position of each servo, $x_{p i}, y_{p i}$. The servo's rotation angles as variant time input variables are determined directly as the outputs of the controller.

As it is noticed, the only solution for swash plate nonlinear and differential equations is the computational one. Since the number of unknowns, the number of swash plate output variables are three variables, $\delta \mathrm{x}, \delta y$ and $\mathrm{zc}$, and they are less than the number of equations which are four equations, the mathematical solution will be trivial one. Newton-Raphson method is used as computational method but the presence of the sinusoidal functions in power forms makes the solution is more complicated and inconsistent during the simulation process, more than one value for each parameter will be resulted for the same used initial values, all of them satisfy the solution of swash plate equations, but some of them are not realistic. To overcome this problem, the solution of swash plate equations of motion is considered to be composed of two partial and separated solutions, for each partial solution, only three equations out of four equations during the simulation process are considered, the omitted equation is considered to that one functions of rotation angle which is supposed to be ignored and equals zero, so the calculation of swash plate longitudinal rotation angle is separated from lateral one, while the total vertical displacement is considered to be the maximum value resulted from both solutions. The resulted values using these assumptions are validated through matching them with the extracted and identified ones from experiment measurements applied on real mini helicopter model [17-18].

The mathematical model of mixer dynamics can be represented by three linear equations of motion, the swash plate output variables will be considered as mixer input variables, while the mixer output variable are represented by rotor blade rotation angle, longitudinal cycling angle $U_{l o n}$, lateral cycling angle $U_{\text {lat }}$ and collective angle $U_{c o l}$ The relationships between swash plate output variables and rotor blade rotation angles are represented mathematically in the following form of the equations.

$$
\begin{gathered}
U_{C o l}=\left[l_{e 7 e 8} /\left(l_{e 3 e 7} l_{e 6 e 8}\right)\right](z c-0.069) \\
U_{\text {Lat }}=r\left[l_{e 7 e 8} /\left(l_{e 3 e 7} l_{e 6 e 8}\right)\right] \delta x \\
U_{\text {Lon }}=-r\left[l_{e 7 e 8} /\left(l_{e 3 e 7} l_{e 6 e 8}\right)\right] \delta y
\end{gathered}
$$

The other variables such as mixer radius $r$ and the lengths of the connecting rods $l_{e 7 e 8}, l_{e 3 e 7}$, and $l_{\text {e6es }}$ have constant values.

\subsection{Flapping Dynamics}

The output variables of mixer mechanism are fed to 
the main rotor blades, each blade is connected to the control rotor via certain mechanical linkage, denoted by $K_{\beta}$, resulting a flapping motion to the main rotor. This motion can be resolved to longitudinal and lateral blade flapping angles $a$ and $b$ respectively. The control moments produced by the flapping motion are the dominant rolling and pitching moments of main rotor. The flapping of the main rotor is described by the TPP(tip-path-plane) equations of motion, these equations are representing the control rotor flapping angel rates in both lateral direction and longitudinal direction as well:

$$
\begin{aligned}
& \dot{\mathrm{a}}=-\mathrm{Q}-\frac{\mathrm{a}}{\tau}+\frac{1}{\tau}\left(\mathrm{A}_{\mathrm{b}} \mathrm{b}+\mathrm{A}_{\text {lon }} \mathrm{U}_{\text {lon }}\right) \\
& \dot{b}=-P-\frac{b}{\tau}+\frac{1}{\tau}\left(B_{a} a+A_{\text {lat }} U_{\text {lat }}\right)
\end{aligned}
$$

where $A_{b}=-B_{a}=\left(\frac{8}{\gamma}\right)\left(\lambda_{\beta}^{2}-1\right)$ is the stiffness number, $\lambda_{\beta}$ is the flapping spring at the hinge, its value is calculated from $\lambda_{\beta}^{2}=1+\left(K_{\beta} / I_{b} \Omega^{2}\right)$ and $\gamma$ is the blade lock number where $\gamma=\left(\rho a_{o} c_{b} \mathrm{R}^{2} / I_{b}\right)$ and $\tau=\left(\frac{16}{\gamma} \Omega^{2}\right) \cdot A_{\text {lon }}$ and $A_{\text {lon }}$ are constants associating the stick command with the blade pitch angle.

Since the small scale helicopter has much faster dynamics than a full sized counterpart and this can pose a problem for the human pilot. This problem is corrected by using a control augmentation in the form of a control rotor called stabilization bar. It consists of a teetering rotor mounted on the same shaft as the main rotor, and made from a steel rod with small paddles at both ends acting as small rotor blades. By teetering rotor it is implied that the control rotor is free to flap without restraint. From the swash plate the control rotor receives longitudinal and lateral inputs, much like the main rotor. But unlike the main rotor it does not receive any collective input, and thus do not produce any lift which would result in a coning angle of the control rotor. It should be noted that mini helicopters generally feature a coupling between the fuselage inertia response and the main rotor flapping response, which is mainly due to the presence of a stabilizer bar.

\subsection{Forces and Moments}

Helicopter dynamics is dominated by the forces and moments generated by the helicopter main rotor and tail rotor additional to the drag and weight forces. The forces and moments originated from the other different elements of a helicopter such as stabilizing bar, fuselage and empennage are considered, while the effects of the forces and the moments originated by the horizontal and vertical stabilizer are neglected. The components of total force and moment $F_{x}, F_{y}, F_{z}$, $L, M$, and Nin body frame are derived from the projection of the main forces and moments acting on miniature helicopter on body frame, these forces and moments are function directly of $U_{c o l}, U_{l o n}, U_{\text {lat }}$ and $U_{p e d}$, and indirectly they are function of servo's rotation angles $\Theta_{i l}$, as they are represented in the following equations [16]:

Main rotor thrust force

$$
\begin{gathered}
\mathrm{T}_{\mathrm{r}}=0.25 \rho \pi \mathrm{R}_{\mathrm{r}}^{2} \Omega_{\mathrm{r}}^{2} \sigma_{\mathrm{r}}\left\{\left[\begin{array}{c}
\mathrm{C}_{\mathrm{l0}}\left(\frac{2}{3}+\mu_{\mathrm{x}}^{2}+\mu_{\mathrm{y}}^{2}\right) \\
\mathrm{C}_{\mathrm{l} \alpha}\left(\mu_{\mathrm{z}}^{2}-\lambda_{0}\right)
\end{array}\right]+\right. \\
\left.\mathrm{C}_{\mathrm{l} \alpha \mathrm{r}}\left[\left(\frac{2}{3}+\mu_{\mathrm{x}}^{2}+\mu_{\mathrm{y}}^{2}\right) \mathrm{U}_{\mathrm{Col}}-\mu_{\mathrm{y}} \mathrm{U}_{\text {lat }}+\mu_{\mathrm{x}} \mathrm{U}_{\text {lon }}\right]\right\}(19)
\end{gathered}
$$

Tail rotor thrust force

$$
\begin{gathered}
\mathrm{T}_{\mathrm{t}}=-0.25 \rho \pi \mathrm{R}_{\mathrm{t}}^{2} \Omega_{\mathrm{t}}^{2} \sigma_{\mathrm{t}} \mathrm{C}_{\mathrm{l \alpha t}}\left\{\mathrm{V}_{\mathrm{t}}-\right. \\
\left.\left[\mathrm{C}_{\mathrm{lo}}\left(\frac{2}{3}+\mathrm{U}_{\mathrm{t}}^{2}+\mathrm{W}_{\mathrm{t}}^{2}\right) \mathrm{U}_{\mathrm{pad}}\right]\right\}
\end{gathered}
$$

Main rotor drag force

$$
\begin{gathered}
Q_{r}=\left(\frac{1}{8}\right) \rho \pi \mathrm{R}_{r}^{5} \Omega_{r}^{2} \sigma_{r} C_{l \alpha}\left\{\left[\begin{array}{c}
\left(\frac{C_{d 0}}{C_{l \alpha}}\right)\left(1+\mu_{x}^{2}+\mu_{y}^{2}\right)- \\
2\left(\mu_{x}-\lambda_{0}\right)^{2}
\end{array}\right]+\right. \\
\left.\left[\left(\lambda_{0}-\mu_{z}\right)\left(\left(\frac{4}{3}\right) U_{\text {col }}-\mu_{y} U_{\text {lat }}+\mu_{z} U_{\text {lon }}\right)\right]\right\}
\end{gathered}
$$

Tail rotor drag force

$$
\begin{gathered}
Q_{t}=\rho \pi \mathrm{R}_{t}^{5} \Omega_{t}^{2} \sigma_{t} C_{l \alpha \mathrm{t}}\left\{( \frac { 1 } { 8 } ) \left[\left(\frac{C_{d 0}}{C_{l \alpha}}\right)\left(1+\mathrm{U}_{t}^{2}+\mathrm{W}_{t}^{2}\right)-\right.\right. \\
\left.\left.2 V_{t}^{2}\right]+\left(\frac{1}{6}\right) \mathrm{V}_{t} U_{\text {pad }}\right\}
\end{gathered}
$$


Main rotor in-plane forces

$$
\begin{gathered}
H r_{x}=\rho \pi \mathrm{R}_{r}^{4} \Omega_{r}^{2} \sigma_{r}\left\{\left[\begin{array}{c}
-0.25 C_{d 0} \mu_{x}+0.125 C_{l \alpha} \\
\left(\mu_{z}-\lambda_{0}\right)\left(2 \mu_{x} U_{C o l}+U_{l o n}\right)
\end{array}\right]\right\}(23) \\
H r_{y}=\rho \pi \mathrm{R}_{r}^{4} \Omega_{r}^{2} \sigma_{r}\left\{\left[\begin{array}{c}
-0.25 C_{d 0} \mu_{y}+0.125 C_{l \alpha} \\
\left(\mu_{z}-\lambda_{0}\right)\left(2 \mu_{y} U_{C o l}-U_{l a t}\right)
\end{array}\right]\right\}
\end{gathered}
$$

Tail rotor in-plane forces

$$
\begin{aligned}
& H t_{x}=-0.25 \rho \pi \mathrm{R}_{t}^{4} \Omega_{t}^{2} \sigma_{t}\left\{C_{d 0} \mathrm{U}_{t}+C_{l \alpha} \mathrm{V}_{t} \mathrm{U}_{t} U_{\text {pad }}\right\} \\
& H t_{z}=-0.25 \rho \pi \mathrm{R}_{t}^{4} \Omega_{t}^{2} \sigma_{t}\left\{C_{d 0} \mathrm{~W}_{t}+C_{l \alpha} \mathrm{V}_{t} \mathrm{~W}_{t} U_{\text {pad }}\right\}
\end{aligned}
$$

Lateral flapping moment

$$
M_{b}=K_{\beta} \sin (b)
$$

Longitudinal flapping moment $M_{a}=K_{\beta} \sin (a)$

Fly bar moment vector

$$
\vec{M}_{f}=\left(-\alpha_{1} U_{l o n}+\alpha_{2} \dot{\Theta}\right) \hat{l}_{b}+\left(\alpha_{3} U_{l a t}+\alpha_{4} \dot{\Phi}\right) \hat{\jmath}_{b}
$$

Where $R$ is the radius of the rotor, $\Omega$ is angular velocity, $c_{l}$ is the blade lift coefficient and $\sigma$ is the rotor solidity factor. $U, V$ and $W$ are the components of the velocity vector. Other terms are calculating as following: $\mu_{x}=\left(U-\mathrm{U}_{w}\right) / \Omega_{r} \mathrm{R}_{r}, \quad \mu_{y}=(V-$ $\left.\mathrm{V}_{w}\right) / \Omega_{r} \mathrm{R}_{r}, \quad \mu_{z}=\left(W-\mathrm{W}_{w}\right) / \Omega_{r} \mathrm{R}_{r}$ and $\lambda_{0}=$ $\mathrm{V}_{i} / \Omega_{r} \mathrm{R}_{r}$, note that the subscript $r$ stands for main rotor while the subscript $t$ stands for tail rotor.

\section{Fuzzy Logic}

Fuzzy logic has rapidly become one of the most successful of today's technologies for developing sophisticated control systems, it has been successfully applied to large numbers of control applications [21]. Fuzzy logic control strategy has shown that it is a good tool for controlling the systems that are difficult to model and it is useful in handling non-linear systems, ill-defined or imprecise problems that depend on the operator skills. In this section, the analysis, design and performance evaluation of the $\mathrm{PD}$ (proportional-derivative) fuzzy logic controller design using Fuzzy Toolbox will be investigated, the proposed controller is applied on full nonlinear mathematical model of miniature helicopter.

\subsection{Fuzzy Controllers}

Fuzzy controllers are more sufficient than classical controllers because they can cover a much wider range of operating conditions, they can be operated with noise and disturbances of a different nature. The method used most often to implement a fuzzy controlleris to use it as a computer program on a general purpose computer; higher density programmable logic devices can be used to integrate large amounts of logic in a single integrated circuit. The main goal of the controller is to stabilize the miniature helicopter around a chosen balanced state vector. In particular, the Euler vector of the stationary state is the main interest.

A PD fuzzy logic controller of the Mamdani type has been be designed, developed and implemented in the flight control system in order to control the miniature helicopter attitudes. The objective of this kind of controllers is to keep the controlled variables at predefined values. Generally, this controller accepts two input signals at each sample time $k$, they are the error $e(k)=x d(k)-x p(k)$, this error signal is obtained by subtracting the real output of the plant $x p$ from the desired output $x d$, and the change of error $\Delta e(k)=e(k)-e(k-1)$, the change of error is obtained by subtracting the previous error signal from the current error signal. The controller output is $\Delta u(k)$, it represents the required change on control action, so the final control action is $u(k)=u(k-1)+\Delta u(k)$, the control action is fed as input of helicopter equations of motion, it is fed at given particular values for $e(k)$ and $\Delta e(k)$. The form of fuzzy logic controller can be formulated as a set of if-then fuzzy rules, the general form is as following.

$$
\begin{gathered}
\text { ifeis } E^{(k)} \text { and } \Delta \text { eis } \Delta E^{(k)} \text { Then } \Delta \text { uis } \Delta U^{(k)}, \\
k=1, \ldots \ldots, n
\end{gathered}
$$

Where, $E^{(k)}, \Delta E^{(k)}$ and $\Delta U^{(k)}$ represent the names of fuzzy sets assigned for error, change of error and 
change of output respectively. Each fuzzy set is represented by membership function, five triangular membership functions for each input/output variable are assigned longwise the universe of discourse, they are shown as in Fig. 3, where $N B$ stands for negative big, $P M$ stands for positive medium, $Z E$ stands for zero, etc. Each variable of the input and output variables can be represented by the generalized fuzzy sets, the universe of discourse for each input and output variable is normalized within the range $[-1 \ldots+1]$ based on its designed scaling factor.

The numbers of rules that govern the controller performance are $5 \times 5=25$ rules, the fuzzy rules relate the controller output to its inputs, and they are summarized in look up table as it is shown in Table 1.

\subsection{Flight Control System Design}

Helicopters have very complicated dynamics, with strong non-linearity and coupling between different control channels. To some extent, the control of small scale helicopters is even more challenging than that of conventional ones since they are more susceptible to ground effects and to the change of both structure and propulsion [22]. An intelligent control algorithm to control the dynamical behavior of miniature helicopter nonlinear mathematical model developed in this research is based on fuzzy logic concepts. The control calculations are performed using m-files in Matlab ${ }^{@}$ environment using standard PC(personal computer) which eases the implementation of calculations and it provides enough computing power for complicated control algorithms such as fuzzy logic toolbox.

The structure of flight control system proposed in this paper is shown in Fig. 4, it is constructed from two main control loops, each one has itsMIMOcontroller, the inner-loop is used for stabilizing the miniature helicopter via adjusting the values of its attitude rates, while the outer-loop is used for tracking the reference values of attitude angles which are given to accomplish the flight mission of miniature helicopter.

\subsubsection{Design of Inner-Loop Controller}

To design the inner-loop controller, the sub controller for each flight dynamic mode of the nonlinear helicopter dynamics will be designed separately based on appropriate assumptions. According to the proposeddesign procedure, the inner-loop control system consistsof three interconnected subsystems. The first subsystem accounts for longitudinal mode and the second subsystem accounts for lateral mode while the third subsystem accounts for directional mode. When the controller for one of the dynamics modes is designed, the control input values for other subsystems are fixed at certain values and vice versa, these values

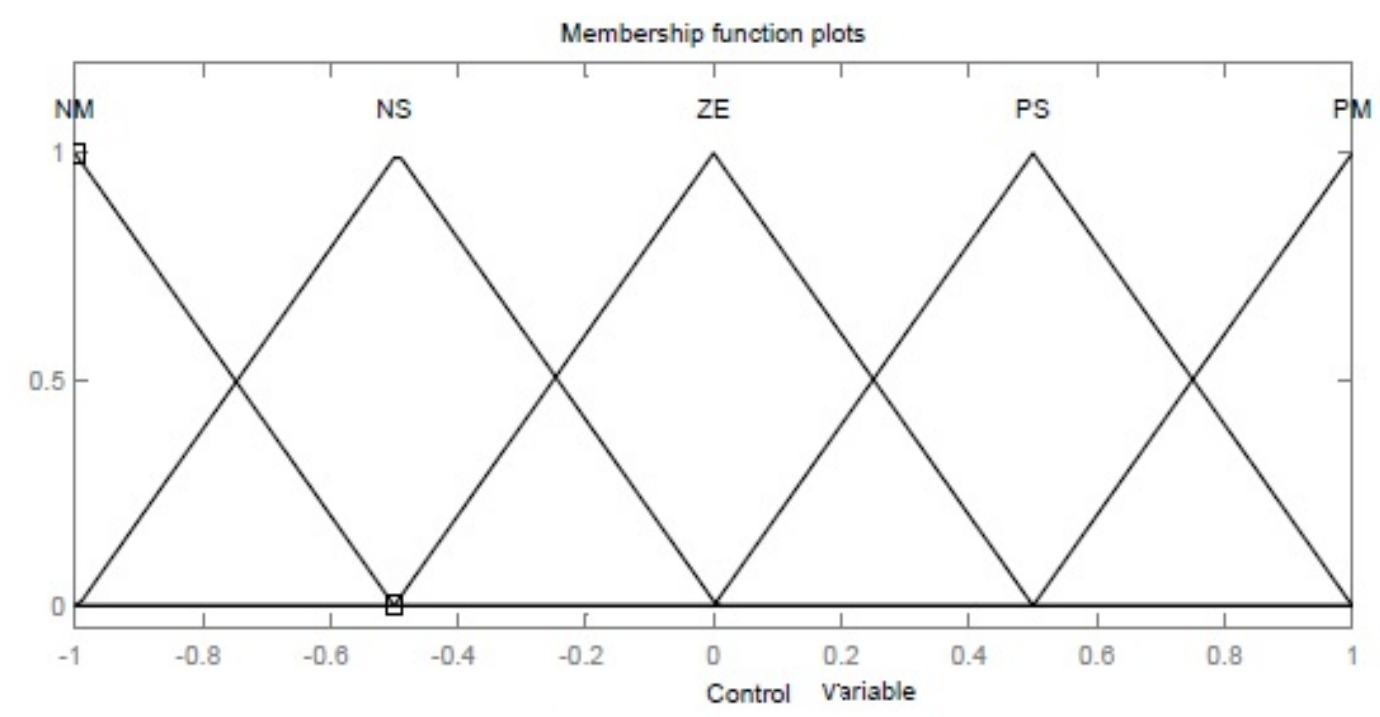

Fig. 3 Generalized fuzzy sets. 
Table 1 Fuzzy rule look up table.

\begin{tabular}{|c|c|c|c|c|c|}
\hline $\mathbf{e}$ & NM & NS & ZE & PS & PM \\
\hline PM & ZE & PS & PM & PM & PM \\
\hline PS & NS & ZE & PS & PM & PM \\
\hline ZE & NM & NS & ZE & PS & PM \\
\hline NS & NM & NM & NS & ZE & PS \\
\hline NM & NM & NM & NM & NS & ZE \\
\hline
\end{tabular}

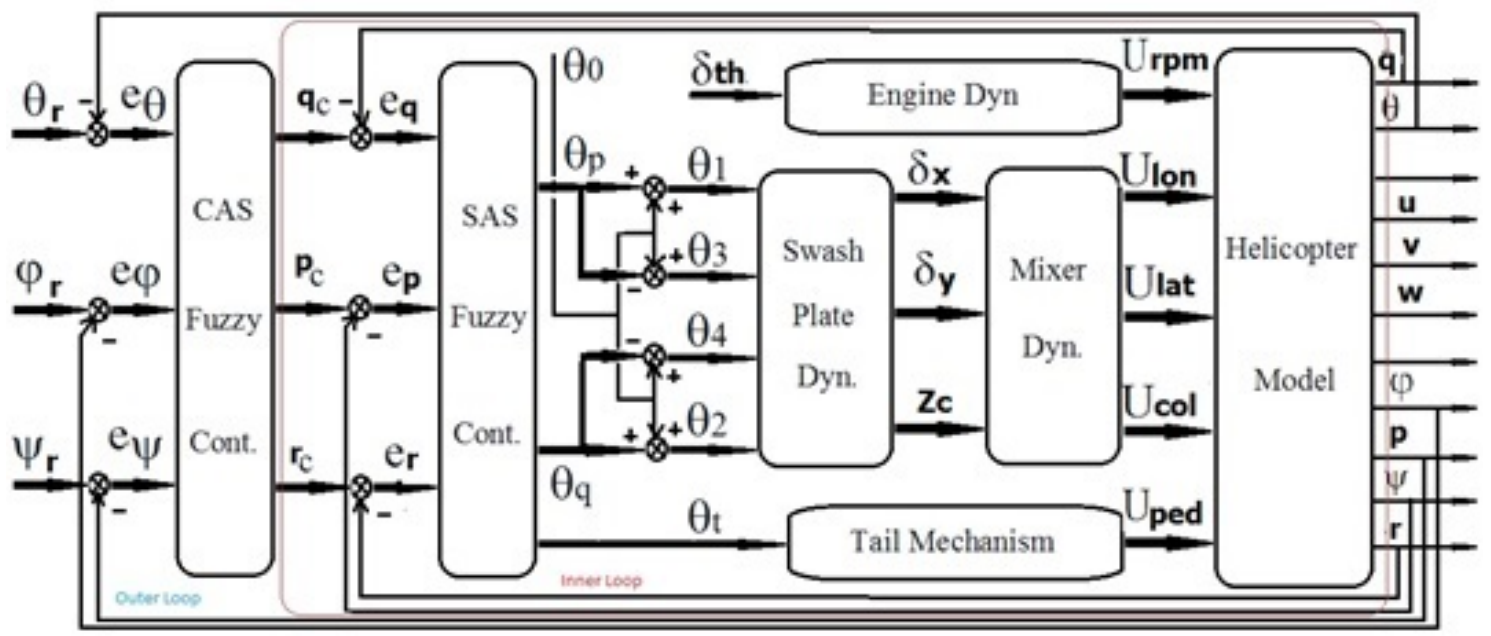

Fig. 4 Block diagram of control system.

are matched with the offset values of the related servo rotation angles which lead to null values of both longitudinal and lateral cycling angles. The time responses of miniature helicopter open loop dynamic when the servo's rotation angles have set to their offset values, $\theta_{1}=-24.50^{\circ}$ for the first servo and $\theta_{2}=17.50^{\circ}$ for the second servo, so the offset rotation angle for the third servo is $\theta_{3}=+24.50^{\circ}$, and the offset rotation angel for the forth servo is $\theta_{4}=-17.50^{\circ}$ are shown in Fig. 5. The offset value of rotation angle for tail servo is $\theta_{t}=-11.50^{\circ}$, while $\theta_{0}$ is the additional angle rotation added simultaneously to all swash plate servo rotation angels in order to control the collective pitch angle.

PD fuzzy controller mounted in forward pass of each control channel is used to adjust the required dynamic of each helicopter dynamic mode, so three MISO sub-controllers in the inner loop of flight control system are designed separately, the designed sub-controllers are blended together to form MIMOfuzzy controller for controlling the inner loop dynamic of helicopter nonlinear mathematical model, the resulted scaling factors from subsystem controllers are retuned in order to produce the desired time responses for rolling rate, pitching rate and yawing rate. The overall scaling factor values for stability augmentation loop controller input variables and control variables are as shown in Table2. The controller outputs of stability augmentation controller will be accumulated with previous control variables to form new control inputs for helicopter mathematical model.

The time responses of stability augmentation control system for unity step inputs are shown in Fig. 6. It is noticed that using PD-MIMO fuzzy controller, the control system has fast time responses with zero steady state errors. 

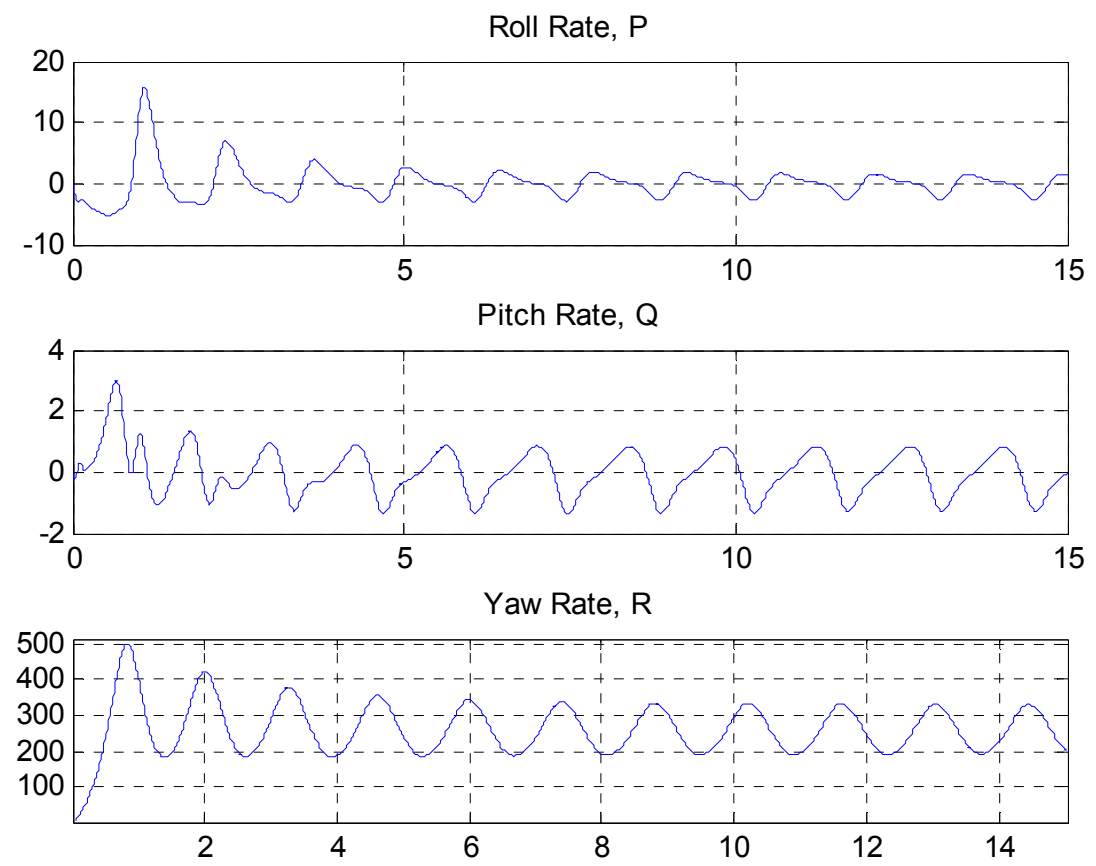

Fig. 5 Time responses of open loop control system.

Table 2 Stability augmentation controller scaling factors.

\begin{tabular}{llll}
\hline Scaling factordynamic mode & Error,E & Change of error, $\Delta \mathrm{E}$ & Output, $\Delta \mathrm{U}$ \\
\hline Longitudinal; Q & 10 & 1 & 5 \\
Lateral; P & 10 & 1 & -0.5 \\
Directional; R & 10 & 1 & 1 \\
\hline
\end{tabular}

\subsubsection{Design of Outer-Loop Controller}

The outer-loop controller is designed to achieve the desired responses of the required attitude angles, Euler angles, in the NED frame. The same procedure done in inner loop controller design will be applied on outer loop controller design. The sub controllers of command augmentation system will be designed separately by assuming all outer-loop channels are decoupled, while the designed blended MIMO controller for the closed inner loop will be considered. For each attitude angle control channel, the subsystem controller is mounted in its forward path, the required related attitude angle is only considered,while the rest attitude angles are omitted by considering to have null values and vice versa, this procedure can be repeated in order to design the controller for adjusting the other attitude angles. PD fuzzy controller is used to design the control system, all three MISOdesigned controllers are blended together in order to form MIMOfuzzy controller for adjusting the helicopter attitude angles. the resulted scaling factors are retuned simultaneously in order to obtain the desired time responses for Euler angle, the overall scaling factor values for input variables and output variables of blended controllers are shown in Table 3, while the steady-state of the controlled variables, Euler angle, are shown in Fig. 7 and time responses for stability augmentation system are shown in Fig. 8. The results prove that using PD-MIMOfuzzy controller enables the helicopter attitude system control to keep the helicopter at desired attitude with fast responses and zero steady state errors.

\section{Simulation and Results}

Flight simulation in aerospace engineering is increasingly being required for the design as it 
Roll Rate, $\mathrm{P}$
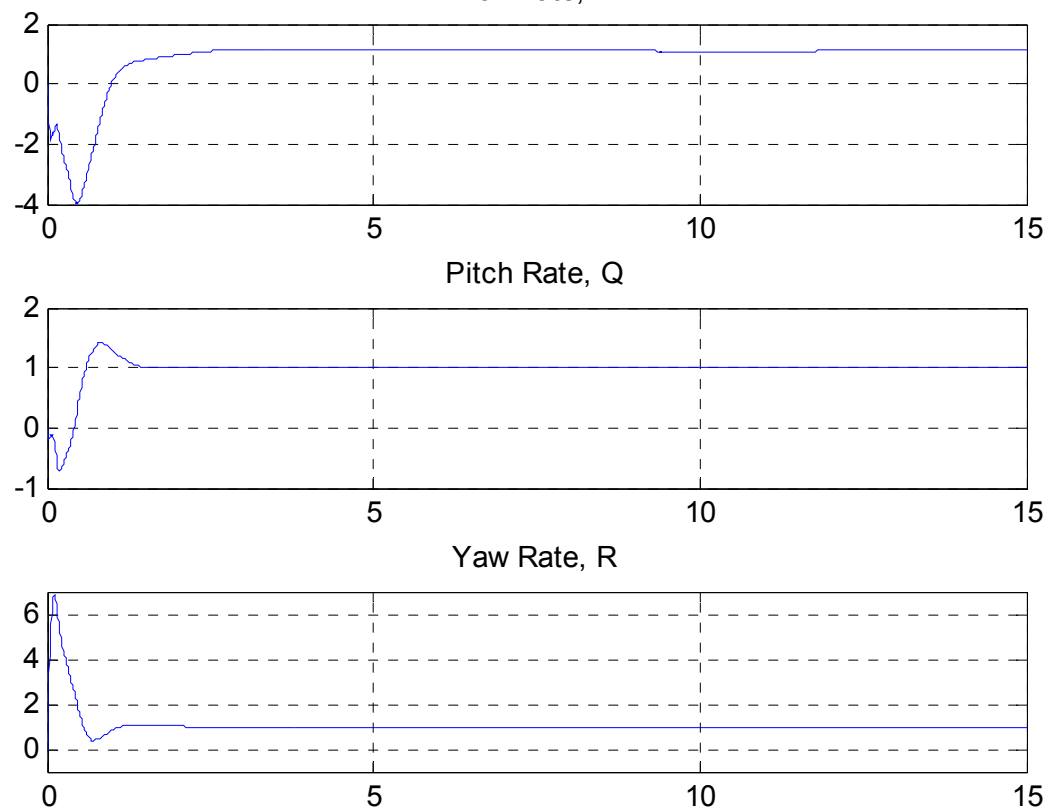

Fig. 6 Stability augmentation system time responses.

Table 3 Command augmentation controller scaling factors.

\begin{tabular}{llll}
\hline Scaling factors & Error, & Change of error, & Output, \\
Attitude angle & $\mathrm{E}$ & $\Delta \mathrm{E}$ & $\Delta \mathrm{U}$ \\
\hline Longitudinal; (Thita) & 10 & 1 & 1 \\
Lateral; (Phi) & 10 & 1 & 1 \\
Directional; (Psi) & 10 & 0.1 & 1 \\
\hline
\end{tabular}

Phi

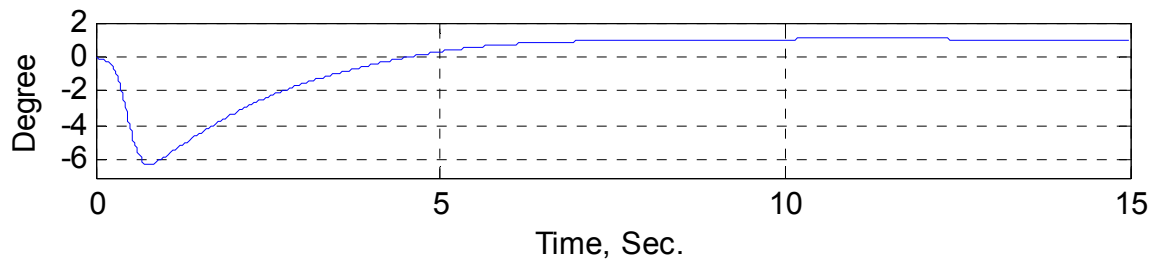

Thi

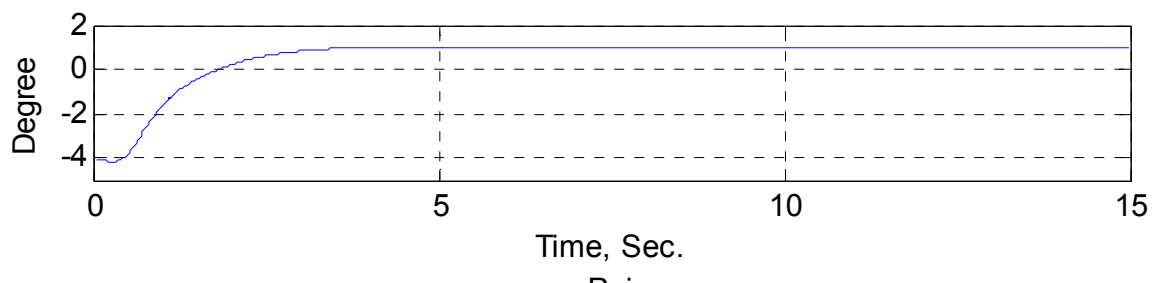

Psi

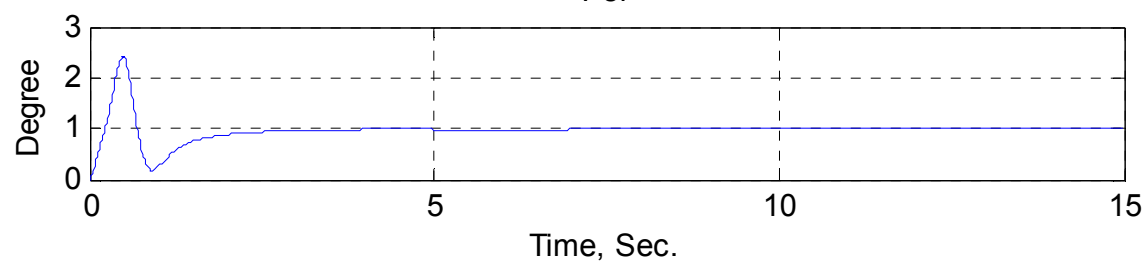

Fig. 7 The step responses of command control systems. 

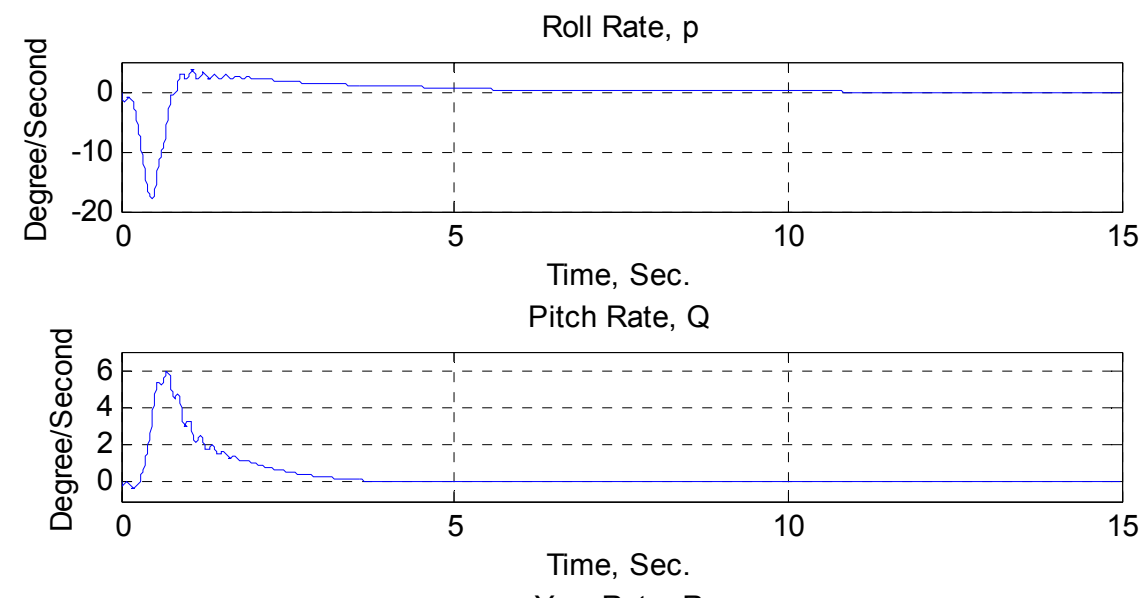

Yaw Rate, R

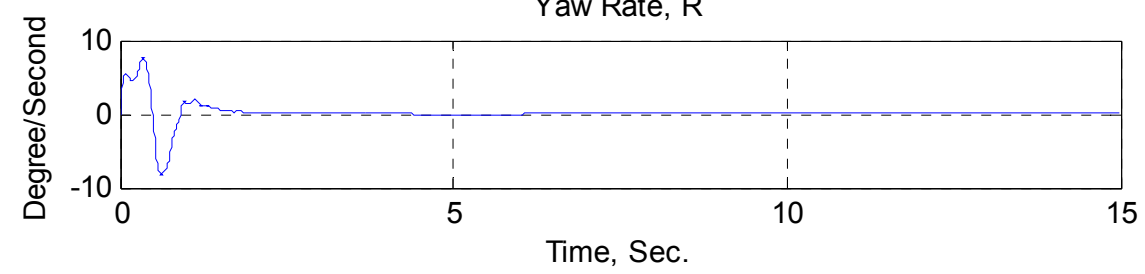

Fig. 8 The time responses of stability augmentation system.

becomes a very beneficial tool for exploring a variety of outcomes without taking a risk of losing any real vehicles in flight tests. The extensive use ofsimulation for this purposed research is generated to tune the flight controllers during flight control system design stages, saving the program several weeks andperhaps a crash of the aerial vehicle in real flight, the simulation is also used as a tool to correct problems uncovered in flight test and to save considerable time as well [23].

In this section, both the simulation and the actual implementation results for the flight control system obtained in the previous sections will be presented, the advantage of this simulation tool is used as test for a successful designed controllers, the real helicopter is required to be replaced by the mathematical helicopter model. Both developed helicopter nonlinear dynamic and fuzzy controller models were simulated in MATLAB environment using $\mathrm{m}$-file programing and fuzzy Toolbox as well. Detailed models for the servos, swashplate and mixer mechanisms and environment data were incorporated in the simulation. Fig. 9 illustrates the simulation results using MATLAB
Simulation program when the desired value for attitude angle as control variables are applied, Fig. 9 depicts the time history for most of important parameters used in control system design process programming, it shows the required rotational angles of all servos, rotational and displacement motions of swash plate, components of main blade pitching angle in addition to lateral and longitudinal flapping angles, actual attitude angle rate and the actual Euler angles.

It is noticed that Fuzzy logic concepts and deigned PDfuzzy logic controllers are able to adjust the helicopter attitude control system for required desired values, the resulted values for main variables are visible and achievable.

\section{Conclusion}

In this paper, an expanded nonlinear mathematical model including the equations of motion of helicopter model, swashplate and mixer dynamics is introduced. Fuzzy controllers for complete attitude flight control system of a small-scale helicopter are designed and tested. The simulation results show that the proposed design of the flight controllers is successful andcapable 

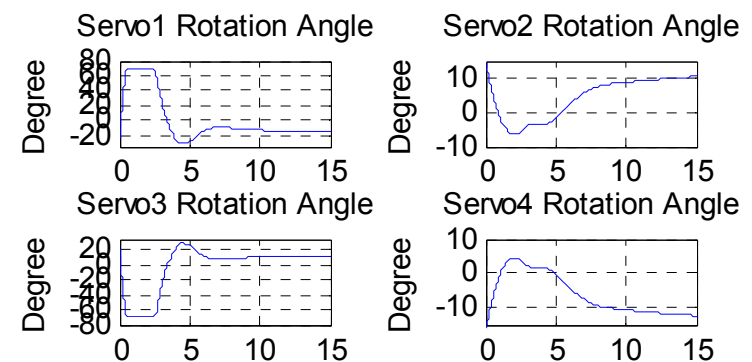

Longitudinal Rotation Angle, Dxateral Rotation Angle, Dy
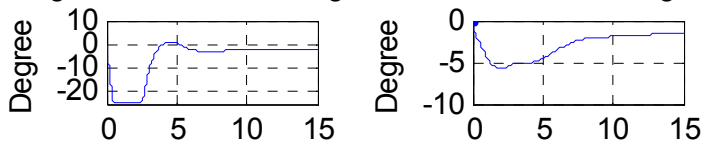

Lateral Pitching Angel

Longitudinal Pitching Angel
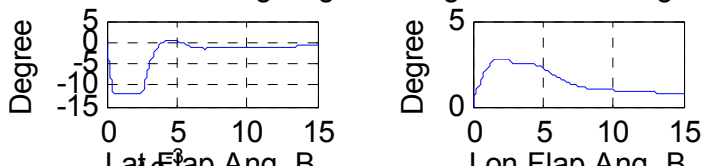

Lał EPap Ang, B

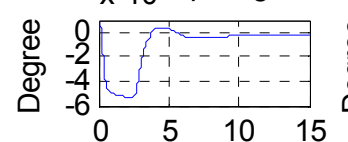

Lon Flap Ang, B
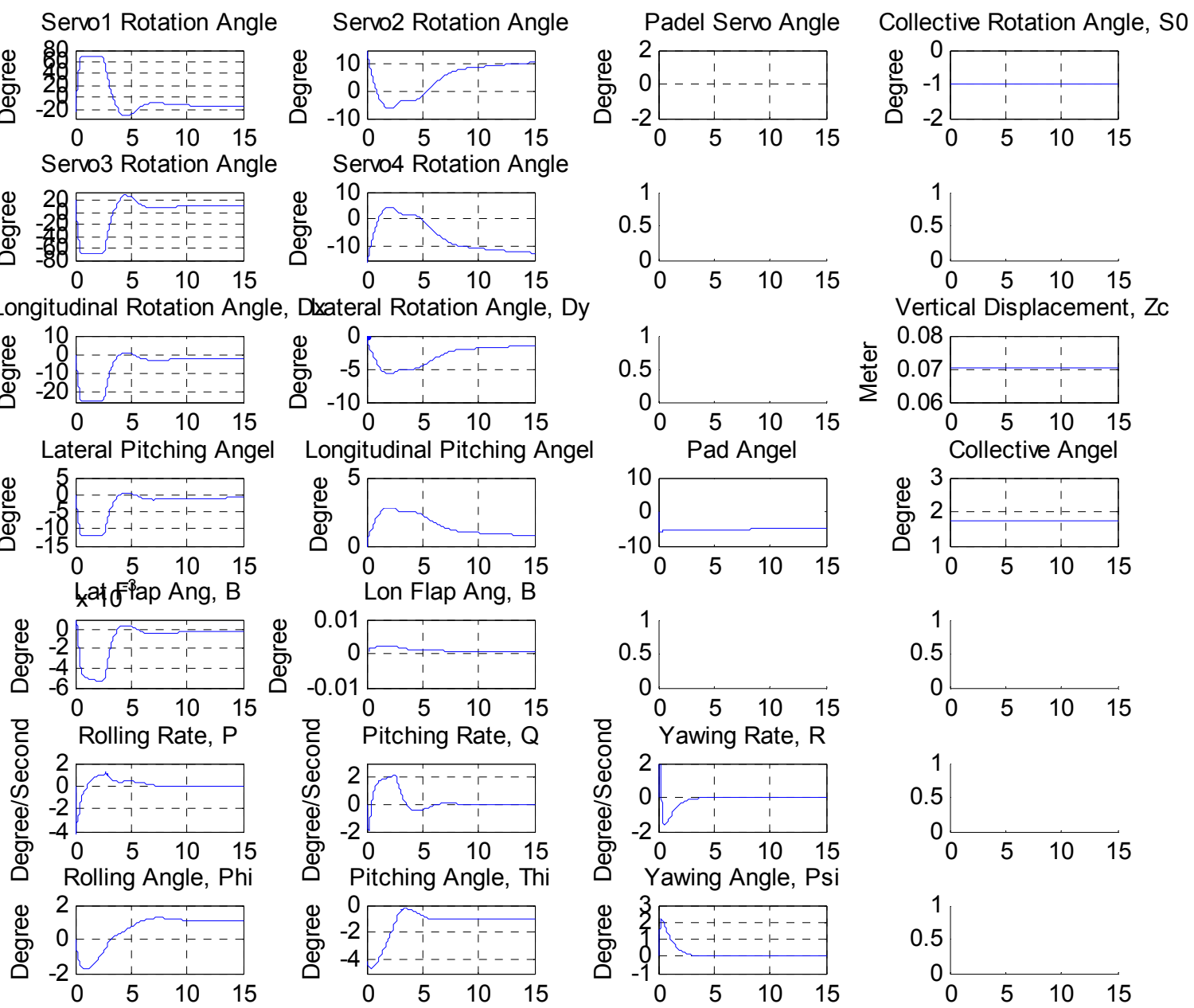

Vertical Displacement, Zc
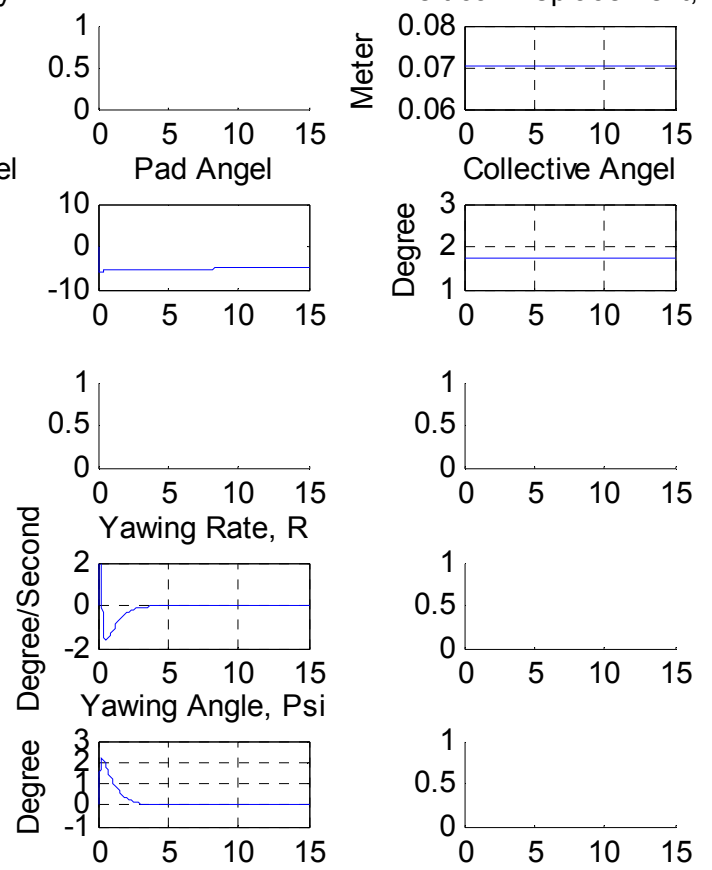

Fig. 9 The time history of control system main variables.

of achieving the top level of performance for the full nonlinear mathematical model of helicopter. The main advantage of this research is designing the attitude control system that be capable to deal with both dynamics of swash plate and mixer within the mathematical model which makes the simulation of helicopter mathematical model closer to the real one. Future work will be considered to implement the developed fuzzy logic controller in real model; the designed controller will be tested, evaluated and tuned on the test bed.

\section{References}

[1] Frazzoli, E., M.Dahleh, and Feron, E. 2002. "Real-time Motion Planning for Agile Autonomous Vehicles." AIAA Journal of Guidance, Control and Dynamics $\quad 25$

\section{(1): 116-29.}

[2] Prouty, R.W.1990.“Helicopter Performance, Stability and Control." Robert E. KriegerPublishing Company, Malabar, Florida.

[3] Langelaan, J.,and Rock, S. 2005. "Navigation of Small UAVs Operating in Forests." In Proc. CD-ROM of AIAA Guidance, Navigation, and Control Conference, San Francisco.

[4] Kim, S. K., and Tilbury, D. M. 2004. "Mathematical Modeling and Experimental Identification of an Unmanned Helicopter Robot with Flybar Dynamics." Journal of Robotic Systems 21 (3): 95-116.

[5] Raptis, I., and Valavanis, K. 2011.Linear and Nonlinear Control of Small-Scale Unmanned Helicopters. Vol. 45. New York: Springer.

[6] Johnson, E.,\& Kannan, K. 2005. “Adaptive Trajectory Control for Autonomous Helicopters." Journal of Guidance, Control and Dynamics 28 (3): 524-38.

[7] Nakwan, K., and Anthony, J. 2004. “Adaptive Output 
Feedback for Altitude Control of an Unmanned Helicopter Using Rotor RPM.” AIAA Paper, 5323.

[8] Hald, U., Hesselbek, M., and Siegumfeldt, M. 2006. "Nonlinear Modeling and Optimal Control of a Miniature Autonomous Helicopter." M.S. thesis, Department of Control Engineering, Aalborg University, Aalborg, Denmark.

[9] Leitner, J., Calise A., and Prasad, J. 1997.“Analysis of Adaptive Neural Networks for Helicopter Flight Controls." AIAA Journal of Guidance, Control, and Dynamics 20 (5): 972-9.

[10] Nakanishi, H., and Inoue, K. 2002. "Development of Autonomous Flight Control Systems for Unmanned Helicopter by Use of Neural Networks." In Proceedings of the 2002 International Joint Conference on Neural Networks, 2626-31.

[11] Apkarian, P., Gahinet, P., and Becker, G. 1995. "Self Scheduled $\mathrm{H}_{\infty}$ Control of Linear Parameter Varying Systems: A Design Example.” Automatica 31(9): 1251-61.

[12] Sugeno, M., Griffin, M., and Bastian, A. 1993"Fuzzy Hierarchical Control of an Unmanned Helicopter." $17^{\text {th }}$ IFSA World Congress, 179-82.

[13] Chao, H., Cao, Y., and Chen, Y.2012. "Autopilots for Small Unmanned Aerial Vehicles: A Survey." International Journal of Control, Automation, and Systems 8(1): 36.

[14] Maiers, J., and Sherif, Y. 1985.“Applicatoins of Fuzzy Sets Theory."IEEE Transactions on System, Man andCybernetics SMC-15 (1):.175-89.

[15] Lee, C. 1990.“Fuzzy Logic in Control Systems: Fuzzy
Logic Controller.'IEEE Transactions on Systems, Man and Cybernetics 20 (2): 404-35.

[16] Gavrilets, V., Mettler, B., and Feron, E. 2001. "Nonlinear Model for a Small-Size Acrobatic Helicopter." In Proceedings of the AIAA Guidance, Navigation, and Control Conference, AIAA 4333.

[17] Khaligh, S. 2014. "Control-Oriented Modeling and System Identification for Nonlinear Trajectory Tracking Control of a Small-Scale Unmanned Helicopter.” Ph.D dissertation, University of Alberta.

[18] Gonzalez, A. 2014. "Instrumentation Design, System Identification, and LQR/LQG Control of a Small Scale Helicopter." M.Sc. thesis, University of Alberta.

[19] Padfield, G. 2007. "Helicopter Flight Dynamics: The Theory and Application of flying qualities and Simulation Modeling." AIAA Education series.

[20] Saffarian, M., and Fahimi, F. 2008. "Comprehensive Kinematic Analysis of a Model Helicopter'S Actuating Mechanism." In Proceedings of 46th AIAA Aerospace sciences meeting and exhibit.

[21] Zhang, L., and Shaojie, B. 2010. "Fuzzy-PID Control Algorithm of the Helicopter Model Flight Attitude Control." IEEE Chinese Control and Decision Conference, Hong Yang.

[22] Barabanov, A., Vazhinsky, N., and Romaev, D. 2007. "Full Autopilot for Small Electrical Helicopter." In Proc. of the 33th European Rotorcraft Forum.

[23] Chen, W., and Wang, D. 2004. "Hardware in the Loop Simulations of an Unmanned Helicopter Flight Control System."Flight Dynamics 22 (1): 41-4. 